\title{
RADIO EMISSIONS FROM THE OUTER HELIOSPHERE
}

D. A. GURNETT and W. S. KURTH

Dept. of Physics and Astronomy, The University of lowa, Iowa City, IA, 52242, USA

\begin{abstract}
For nearly fifteen years the Voyager 1 and 2 spacecraft have been detecting an unusual radio emission in the outer heliosphere in the frequency range from about 2 to $3 \mathrm{kHz}$. Two major events have been observed, the first in 1983-84 and the second in 1992-93. In both cases the onset of the radio emission occurred about 400 days after a period of intense solar activity, the first in mid-July 1982, and the second in May-June 1991. These two periods of solar activity produced the two deepest cosmic ray Forbush decreases ever observed. Forbush decreases are indicative of a system of strong shocks and associated disturbances propagating outward through the heliosphere. The radio emission is believed to have been produced when this system of shocks and disturbances interacted with one of the outer boundaries of the heliosphere, most likely in the vicinity of the the heliopause. The emission is believed to be generated by the shock-driven Langmuir-wave mode conversion mechanism, which produces radiation at the plasma frequency $\left(f_{p}\right)$ and at twice the plasma frequency $\left(2 f_{p}\right)$. From the 400 -day travel time and the known speed of the shocks, the distance to the interaction region can be computed, and is estimated to be in the range from about 110 to $160 \mathrm{AU}$.
\end{abstract}

Key words: Heliospheric Radio Emissions, Heliosphere, Heliopause, Termination Shock

Abbreviations: PWS-Plasma Wave Subsystem, AU-Astronomical Unit, DSN-Deep Space Network, NASA-National Aeronautics and Space Administration, GMIR-Global Merged Interaction Region, MHD-Magnetohydrodynamic, CME-coronal mass ejection, $f_{p}$-plasma frequency, R-radial distance, AGC-automatic gain control

\section{Introduction}

The Voyagers 1 and 2 spacecraft both include an instrument called the Plasma Wave Subsystem (PWS) that can detect the electric field of plasma waves and low-frequency radio emissions. The Voyager plasma wave instrument was originally designed to study plasma waves in the magnetospheres of the outer planets (Scarf and Gurnett, 1977). However, because of an unexpected discovery, the plasma wave instrument is also providing important information on the structure of the outer heliosphere. On August 30, 1983, the PWS on Voyager 1 began detecting a weak radio emission in the frequency range around $3 \mathrm{kHz}$, slightly above the local solar wind plasma frequency (Kurth et al., 1984). At that time Voyager 1 was at a heliocentric radial distance of 17.9 Astronomical Units (AU). Subsequent investigations revealed that the radio emission could also be detected by Voyager 2, which was closer to the Sun, at a heliocentric radial distance of $12.7 \mathrm{AU}$. Since the same emission was being detected at two widely different locations, it was immediately recognized that the signal was most likely of heliospheric origin. However, it was difficult to rule out other potential sources, such as Jupiter. Subsequent observations over a period of nearly fifteen years now show that the radio emission is almost certainly generated in the outer regions of the heliosphere. The purpose of this paper is to summarize the current state of understanding of the heliospheric $2-3 \mathrm{kHz}$ radio emissions. The presentation is organized into five sections. Section 2 discusses an overview of the radio spectrum observations, Section 3 discusses the relationship to intense solar events, Section 4 discusses the radio emission mechanism, Section 5 discusses the radial variation of the plasma frequency, and Section 6 discusses the interpretation of the spectrum.

Space Science Reviews 78: 53-66, 1996.

(c) 1996 Kluwer Academic Publishers. Printed in the Netherlands. 


\section{Overview of the Radio Spectrum Observations}

To give an overview of the observations, Figures 1 and 2 show spectrograms of the radio emission intensities detected by Voyagers 1 and 2 over a fourteen-year period, from January 1,1982 , to December 31,1995 . These spectrograms were produced using data from the PWS wideband receiver. The wideband receiver provides 4-bit samples of the electric field waveform at a sample rate of $28,800 \mathrm{~s}^{-1}$. To produce the spectrograms, the waveform is Fourier transformed with a resolution of approximately $28 \mathrm{~Hz}$. The color in each vertical line of the spectrogram represents a 15-second average of the intensities from the Fourier transforms. The time resolution is determined by the receiving capability of the NASA Deep Space Network (DSN) and varies from as low as one spectrum per month, to as high as two spectrums per week. The short vertical bars at the top of each spectrogram indicate when the individual spectrums were obtained. A color bar indicating the relative intensity is shown above each spectrogram. The dynamic range is $6 \mathrm{~dB}$ from the lowest intensity (blue) to the highest intensity (red). Relative intensity is shown because the wideband receiver utilizes an automatic gain control (AGC), which makes it difficult to determine absolute intensities.

Two periods of unusually intense radio emission activity can be seen in Figures 1 and 2; the first in 1983-84 (Kurth et al., 1984) and the second in 1992-93 (Gurnett et al., 1993). In addition, several somewhat weaker events have been observed, one in late 1985, one in 1989, three in 1990-91 (Kurth et al., 1987; Kurth and Gurnett, 1991), and several immediately after the 1992-93 event. By comparing the two spectrograms, it is evident that the radio emission spectrums detected by the two spacecraft are nearly identical, particularly during the later years when the spacecraft are farther from the Sun. During the 1983-84 event, one can see that the spectrum extends to lower frequencies at Voyager 1 than at Voyager 2 . This difference in the spectrum is believed to be due to the propagation cutoff at the local electron plasma frequency, $f_{p}=9 \sqrt{n} \mathrm{kHz}$, where $n$ is the electron number density in $\mathrm{cm}^{-3}$. Since the electron density in the solar wind varies as $1 / \mathrm{R}^{2}$, where $\mathrm{R}$ is the radial distance from the Sun, the solar wind plasma frequency is expected to vary as 1/R. Based on the plasma measurements of Belcher et al. (1993), the plasma frequency at Voyager 2, which was at $\mathrm{R} \simeq 13 \mathrm{AU}$, has an average value of $1.6 \mathrm{kHz}$, with peaks extending up to about $3.7 \mathrm{kHz}$. In contrast, the plasma frequency at Voyager 1 , which was farther from the Sun $(\mathrm{R} \simeq 18 \mathrm{AU})$, is estimated to have an average value of only $1.1 \mathrm{kHz}$, with peaks extending up to about $2.5 \mathrm{kHz}$. Thus, the radiation could reach Voyager 1 at a lower frequencies than for Voyager 2, which explains the difference in the low-frequency cutoffs for the two events.

Simple inspection of the spectrums during both the 1983-84 and 1992-93 events shows that the radio emission has two components, the first consisting of narrow bands that drift upwards in frequency from about 2.0 to $3.6 \mathrm{kHz}$ at rates that vary from about 1 to $3 \mathrm{kHz} / \mathrm{year}$, and the second consisting of a band of emission around $2 \mathrm{kHz}$ that persists for time scales on the order of one year or more. The onset of an upward drifting band is often associated with an intensification of the $2-\mathrm{kHz}$ band. Diffuse structures can also be seen in the $2-\mathrm{kHz}$ band, sometimes drifting slowly upward in frequency. During the $1992-$ 95 period, the $2-\mathrm{kHz}$ band has a very sharp low-frequency cutoff at $1.8 \mathrm{kHz}$. This cutoff cannot be due to a local propagation cutoff, since the $1 / \mathrm{R}$ dependence shows that the local plasma frequency during the $1992-95$ period should be only a few hundred $\mathrm{Hz}$. It is also evident that the 1992-93 event is much more intense than the 1983-84 event, particularly for the $2-\mathrm{kHz}$ band. The higher intensities during the $1992-93$ event are attributed to the lower plasma frequencies compared to the 1983-84 event, which allows a broader range of frequencies to reach the spacecraft. By using absolute intensity measurements from the 


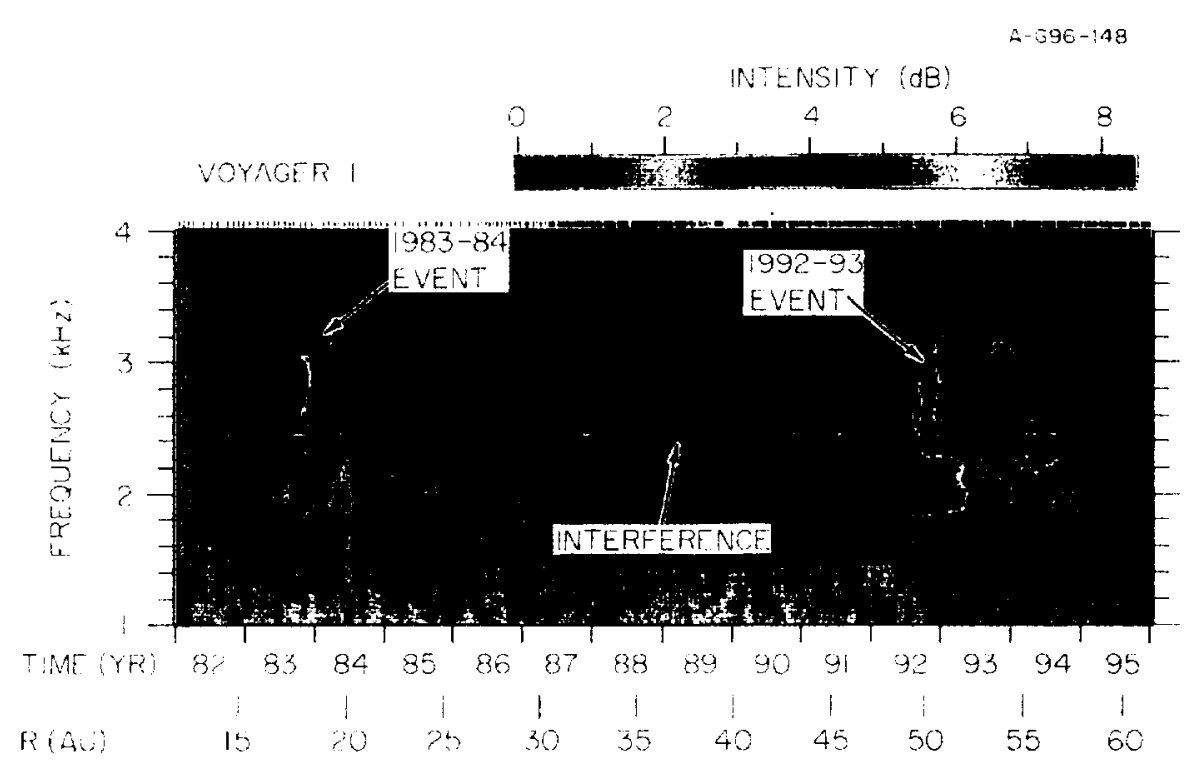

Figure 1. A 14-year frequency-time spectrogram showing the heliospheric radio emissions detected by Voyager 1. Two unusually strong events have been observed, the first in 1983-84 and the second in 1992-93, as well as a number of weaker events. The strong line at $2.4 \mathrm{kHz}$ is interference from the spacecraft power system.

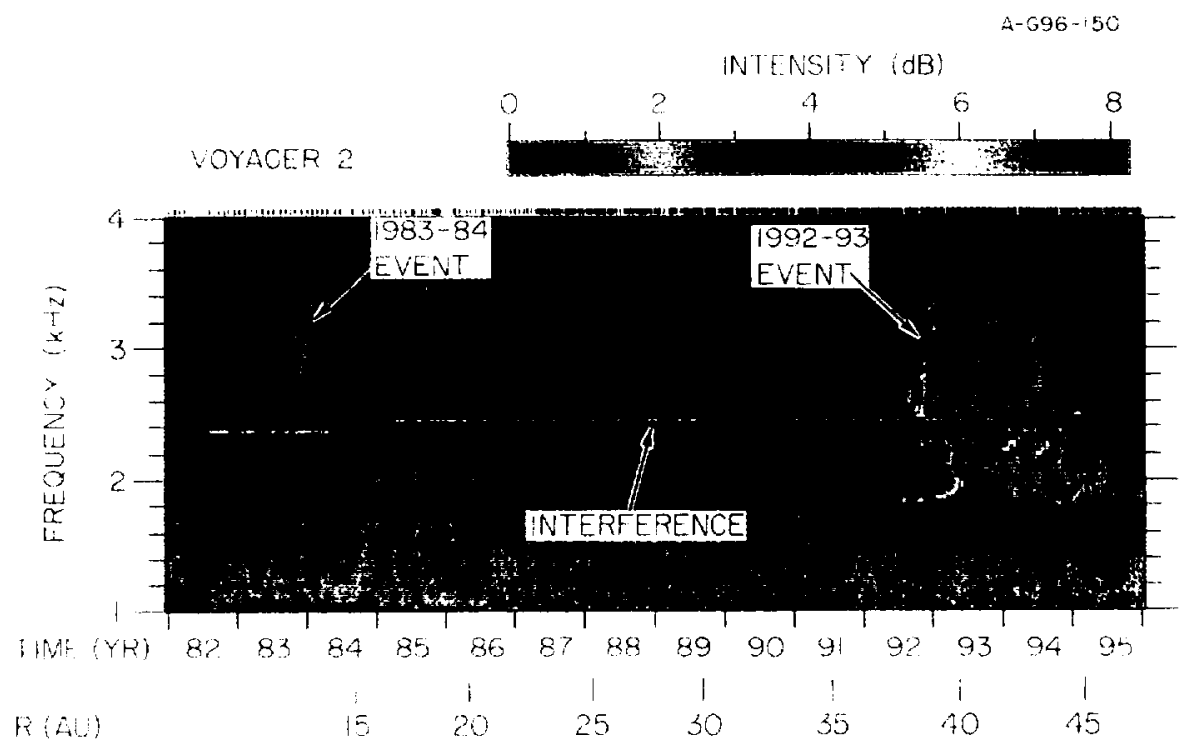

Figure 2. A 14-year spectrogram from Voyager 2 similar to Figure 1. Although separated by distances as large as $50 \mathrm{AU}$, the radio emission spectrums from the two spacecraft are remarkably similar, which shows that the source is at a considerable distance from the spacecraft, probably more than $50 \mathrm{AU}$. 
PWS on-board spectrum analyzer, which has discrete channels at 1.78 and $3.11 \mathrm{kHz}$, Gurnett et al. (1993) estimated that the total radiated power for the 1992-93 event was at least $10^{13}$ Watts. This power level ranks the heliospheric $2-3 \mathrm{kHz}$ radio emissions as one of the strongest radio sources in the solar system, considerably more intense than the strongest planetary radio sources, and comparable to the most intense solar radio bursts.

\section{Relationship to Intense Solar Events}

Since the heliospheric $2-3 \mathrm{kHz}$ radio emission clearly occurs in distinct bursts, typically lasting a fraction of a year or longer, the question naturally arises as to what "triggers" these bursts. McNutt (1988) was the first to suggest that solar wind disturbances caused by transient activity at the Sun could trigger a burst of $2-3 \mathrm{kHz}$ radio emission. In particular, he proposed that the 1983-84 radio emission event was produced by the interaction of a high-speed solar wind stream with the termination shock. This idea was further explored by Grzedzielski and Lazarus (1993), who identified a series of dynamic pressure increases in the solar wind that they believed were responsible for the 1983-84, 1985, and 1989 events, again assuming an interaction with the termination shock. Despite the merit of these ideas, the time delay between candidate solar wind transients and the onset of the radio bursts varied over such a wide range that the hypothesized cause-effect relationship was not convincing.

With the onset of the intense 1992-93 radio emission event, the evidence for a solar wind trigger improved significantly. This event was so intense that one would expect that it should be associated with an extraordinary solar event. Indeed, an extraordinary event was soon found, namely, the great cosmic ray Forbush decrease of 1991 (Gurnett et al., 1993), which occurred about 400 days before the onset of the radio emission. In the process of reviewing the earlier data, it was soon discovered that the intense 1983-84 radio emission event was also preceded in 1982 by an extraordinarily large Forbush decrease, again about 400 days before the onset of the radio emission. These relationships are illustrated in Figure 3 , which shows the cosmic ray intensity from the Deep River neutron monitor in the top panel and the radio emission intensity from the $3.1-\mathrm{kHz}$ channel of the Voyager 1 spectrum analyzer in the bottom panel. The two large Forbush decreases, labelled A and B, were produced by periods of intense solar activity in mid-July 1982 and May-June 1991, during the declining phases of solar cycles number 21 and 22 . These two Forbush decreases are the two deepest Forbush decreases ever observed ( $21 \%$ and $30 \%$, respectively). As can be seen, the 1983-84 radio emission event (labelled $A^{\prime}$ ) started about 412 days after Forbush decrease $A$, and the 1992-93 radio emission event (labelled $B^{\prime}$ ) started about 419 days after Forbush decrease B.

The current view is that large Forbush decreases, such as events A and B, are caused by a series of outward propagating solar wind disturbances that merge in the outer heliosphere to form a shell of compressed plasma and magnetic field called a Global Merged Interaction Region (GMIR). For a discussion of GMIRs, see Burlaga et al. (1993) and McDonald and Burlaga (1996). GMIRs are usually preceded by a strong leading shock that is formed by the coalescence of several shocks, each of which originates from a specific event at the Sun. The strong leading shock is typically followed by a region of turbulent plasma with numerous magnetohydrodynamic (MHD) discontinuities. Other shocks may also be imbedded within the GMIR. The strong turbulent magnetic fields in the GMIR scatter and impede the transmission of cosmic rays, thereby causing the transient cosmic ray intensity decreases known as Forbush decreases.

The Forbush decreases associated with events A and B were observed by a number of interplanetary spacecraft (Van Allen and Randall, 1985; Webber et al., 1986; Cliver et al., 


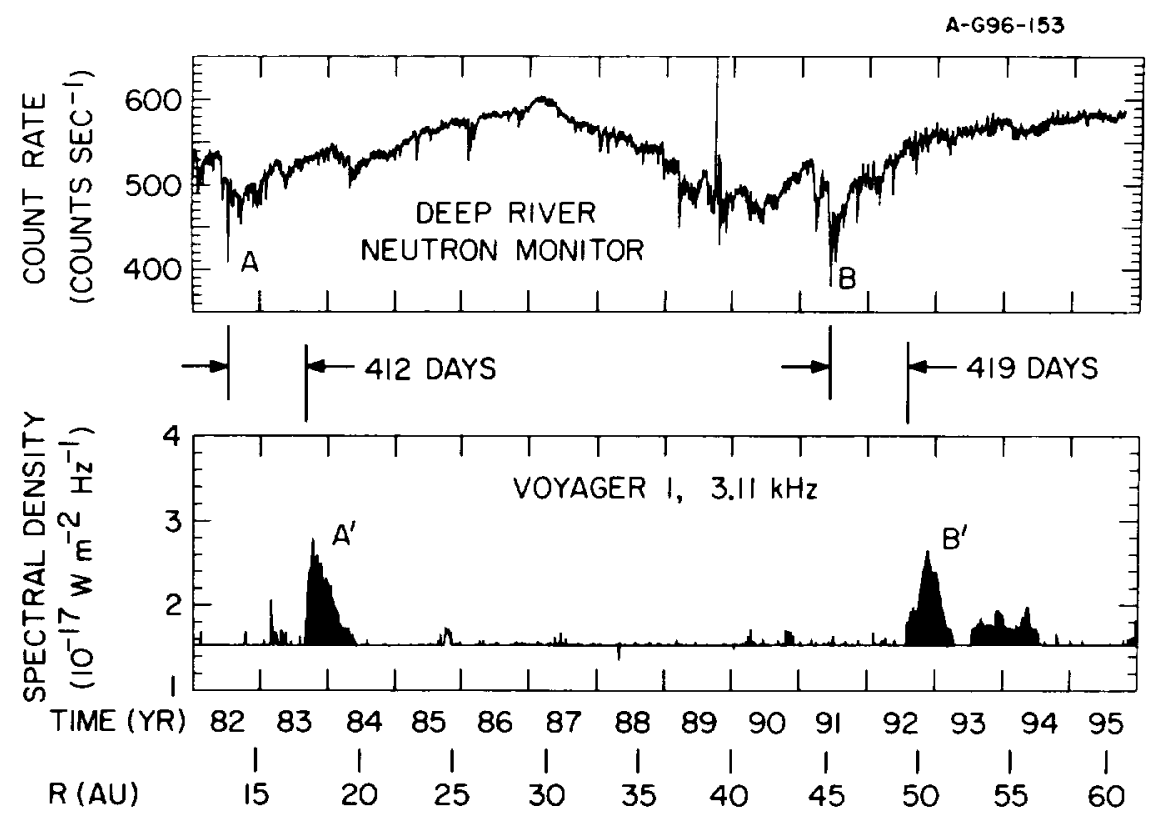

Figure 3. A 14-year plot showing the radio emission intensity at $3.11 \mathrm{kHz}$ from Voyager 1 , and the corresponding cosmic ray intensity from the Deep River neutron monitor. The Forbush decreases marked $A$ and $B$ are the two deepest depressions ever observed. The two strong heliospheric radio emission events marked $A^{\prime}$ and $B^{\prime}$, occurred about 400 days after these large Forbush decreases.

1987; Van Allen and Fillius, 1992; Webber and Lockwood, 1993; McDonald et al., 1994). Since both events are rather similar, we focus our attention on event $B$, which has the best coverage at large heliocentric distances. The top panel of Figure 4 shows an expanded plot of the count rate from the Deep River neutron monitor around the time of event $B$. The next four panels show the cosmic ray intensities from Pioneers 10 and 11 , and from Voyagers 1 and 2. Clearly defined Forbush decreases can be seen at all four spacecraft, first by Pioneer 10 on day 233 at $34 \mathrm{AU}$, then by Voyager 2 on day 250 at $35 \mathrm{AU}$, then by Voyager 1 on day 257 at $46 \mathrm{AU}$, and finally by Pioneer 10 on day 273 at $53 \mathrm{AU}$. A strong leading shock was also observed coincident with these Forbush decreases by the Pioneer 10 magnetometer on day 232 (Winterhalter et al., 1992), by the Voyager 2 plasma instrument on day 251 (Belcher et al., 1993), and by the Voyager 1 plasma wave instrument on day 257 (Kurth and Gurnett, 1993).

Two periods of solar activity potentially contributed to the interplanetary shocks and associated cosmic ray decreases illustrated in Figure 4. The first, labelled the March solar events, consisted of 35 solar flares of classification M-5, or higher, that occurred in the southern hemisphere of the Sun from March 1 through March 31, 1991 (McDonald et al., 1994). These events caused the well-defined Forbush decrease in the Deep River neutron monitor on day 83 (March 24, 1991). The second, labelled the May-June solar events, consisted of an even more intense period of solar activity in the northern hemisphere about 6 weeks later, from May 25 to June 15, 1991. During this period, a total of $70 \mathrm{M}$-class and $6 \mathrm{X}$-class solar flares were observed (McDonald et al., 1994). This extraordinarily intense activity produced a sharp decline in the Deep River neutron monitor, causing the deep (30\%) Forbush decrease labelled event B, which occurred on day 163 (June 12), 1991. 


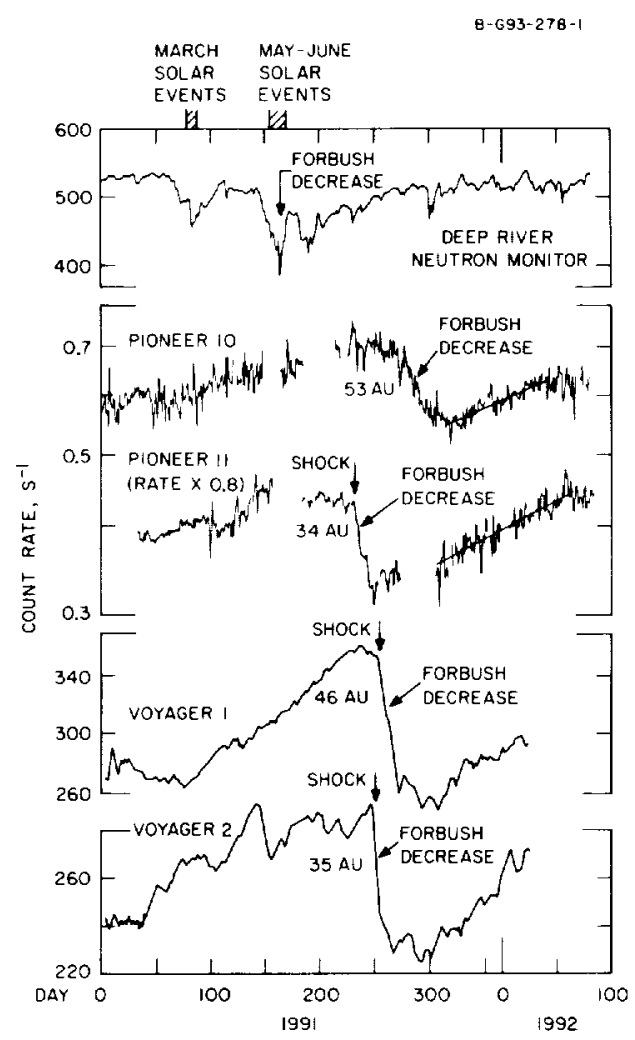

Figure 4. The cosmic ray intensities from Pioneers 10 and 11, and Voyagers 1 and 2, showing the large Forbush decreases produced by the May-June 1991 solar activity. This illustration was adapted from Van Allen and Fillius (1992) and Webber and Lockwood (1993).

The radial propagation velocity that one infers from these observations depends on which spacecraft are used and on which period of solar activity is assumed to be the causative event. Van Allen and Fillius (1992) and Webber and Lockwood (1993) both conclude that the May-June solar activity was primarily responsible for the cosmic ray decreases observed by Pioneers 10 and 11 and Voyagers 1 and 2. Initially, Van Allen and Fillius estimated the propagation speed of this event to be $820 \mathrm{~km} / \mathrm{s}$, but later, based on a more extensive analysis, Van Allen (1993) revised the best fit propagation speed to $865 \pm 75$ $\mathrm{km} / \mathrm{s}$. Webber and Lockwood quote two speeds, 580 and $740 \mathrm{~km} / \mathrm{s}$, depending on whether the time delay is computed between Earth and Voyager 2 or Earth and Voyager 1. McDonald et al. (1994), on the other hand, assumed that the March solar activity was responsible for the Forbush decreases observed in the outer heliosphere. Their estimate of the propagation speed was somewhat lower, $572 \mathrm{~km} / \mathrm{s}$.

In order to visualize how these events relate to the 1992-93 heliospheric radio emission event, it is useful to show all of these events on the same time scale. Such a plot is given in Figure 5 (from Gurnett and Kurth (1995) who also show a similar plot for event A). The top panel shows the count rate from the Deep River neutron monitor, and the bottom two panels show the radio emission intensity from the 1.78 - and $3.11-\mathrm{kHz}$ channels of the Voyager 1 spectrum analyzer. The times of the March and May-June solar events, and the 


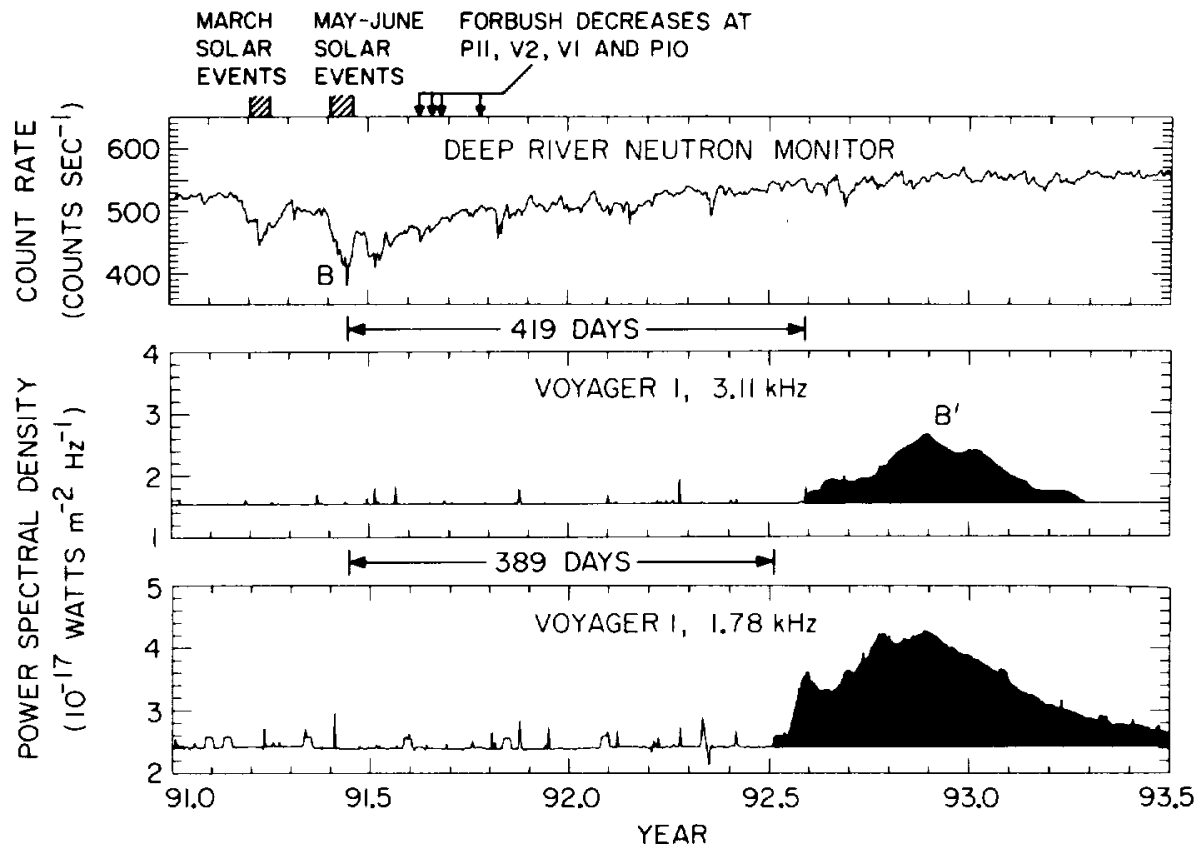

Figure 5. An expanded time scale plot showing further details of events $\mathbf{B}$ and $\mathbf{B}^{\prime}$. The deep Forbush decrease in 1991 was caused by two periods of intense solar activity, the first in March, and the second in May-June. The times at which this Forbush decrease was detected by Pioneer 11, Voyager 2, Voyager 1, and Pioneer 10 (see Figure 4) are shown at the top of the plot. The bottom two panels show the radio emission intensities at 1.78 and $3.11 \mathrm{kHz}$.

onset times of the Forbush decreases at Pioneers 10 and 11 , and Voyagers 1 and 2 are shown at the top of the plot. As one can see, the onset time of the radio emission event in the $1.78-\mathrm{kHz}$ channel occurred 389 days after Forbush decrease B, just a few days before the onset in the $3.11-\mathrm{kHz}$ channel. If the propagation speed is assumed to be independent of distance from the Sun, then the radial distance to the radio emission source, $\mathbf{R}_{S}$, can be computed by using the simple proportion, $R / \Delta t=R_{S} / 389$, where $R$ is the radial distance to the spacecraft, and $\Delta t$ is the time delay from the event on the Sun to the onset of the Forbush decrease at that spacecraft. Using this formula, the distances to the source using the onset times for the Forbush decreases at Pioneers 10 and 11 and Voyagers 1 and 2, work out to be $187,188,190$, and $156 \mathrm{AU}$. This simple calculation shows that the radio emission source is at a considerable distance from the Sun, well beyond $100 \mathrm{AU}$. Of course, the propagation speed most likely decreases in the outer regions of the heliosphere. The effect of the decreasing propagation speed with increasing radial distance has been studied by several investigators, including Gurnett et al. (1993), Steinolfson and Gurnett (1995), Gurnett and Kurth (1995), McNutt et al. (1995), and Liewer et al. (1996). These studies show that the distance to the source is most likely in the range from about 110 to $160 \mathrm{AU}$. Similar results have been obtained for the 1983-84 radio emission event (see Gurnett and Kurth (1995)). 


\section{Radio Emission Mechanism}

Before one can attempt to interpret the radio emission spectrum, it is first necessary to understand the mechanism by which the radio emission is generated. Most of the wellknown astrophysical radio emission mechanisms, such as synchrotron radiation and cyclotron maser radiation, require relatively strong magnetic fields. However, the magnetic field in the outer heliosphere is believed to be extremely weak, $1 \mathrm{nT}$ or less (Axford, 1990). In the weak magnetic field regime only one mechanism is known that could account for the heliospheric radio emissions, namely, the shock-driven Langmuir-wave mode conversion mechanism (Cairns and Gurnett, 1992).

To explain how this radio emission mechanism works, it is useful to discuss type II solar radio bursts, which are believed to be generated by the same mechanism. Type II solar radio bursts are produced by coronal mass ejections (CMEs), which are transient ejections of material from the Sun. CMEs are typically accompanied by solar flares and other forms of solar activity (Gosling, 1993). As the ejected material from the CME moves outward from the Sun, it acts as a piston, which drives a shock ahead of the CME. Strong electric fields within the shock accelerate a beam of electrons outward along magnetic field lines ahead of the shock as shown in Figure 6. This beam generates electrostatic oscillations called Langmuir waves, which in turn decay into electromagnetic radiation via a nonlinear mode coupling process. Typically electromagnetic radiation is generated at two frequencies: the plasma frequency, $f_{p}$, and twice the plasma frequency, $2 f_{p}$. The radiation at $f_{p}$ is called the fundamental, and the radiation at $2 f_{p}$ is called the harmonic. The decrease in the plasma frequency with increasing distance from the Sun causes the emission frequency to decrease as the shock propagates outward from the Sun, thereby producing the characteristic signature of a type II radio burst, which is an emission frequency that decreases with increasing time. The reason that the radiation is generated at both the fundamental and the harmonic is complicated, and has been the subject of numerous theoretical analyses. For a review of the mechanisms involved, see Melrose (1985).

\section{Radial Variation of the Plasma Frequency}

Since strong shocks are known to be propagating through the outer regions of the heliosphere in response to both the July 1982, and May-June 1991 events, it is clear that the proper conditions are present for generating the heliospheric radio emissions via the shockdriven Langmuir-wave mode conversion mechanism. The basic mechanism would then be similar to a type II solar radio burst. However, there are several important differences. Whereas the intensity of a type II radio burst varies in a smooth and continuous manner as the shock propagates outward from the Sun, the heliospheric radio emission events all have sudden onsets. Since no radio emission was detected as the shock propagated past the spacecraft, the sudden onset strongly suggests that the shock has encountered a plasma with significantly different properties, such as would occur at one of the outer boundaries of the heliosphere. There are two such boundaries, the termination shock and the heliopause. To decide which boundary is involved, we must consider the radial variation of the plasma frequency, since the emission must be generated at either $f_{p}$ or $2 f_{p}$.

As discussed earlier, in the region of supersonic flow the solar wind plasma frequency varies inversely with distance from the Sun. On a $\log$ - $\log$ plot, the $f_{p}$ versus R profile is then a straight line with a slope of minus one, as shown in Figure 7. Given the large distances to the interaction region implied by the delay times and shock propagation speeds discussed earlier, it is reasonable to assume that the termination shock is located at about $100 \mathrm{AU}$. 

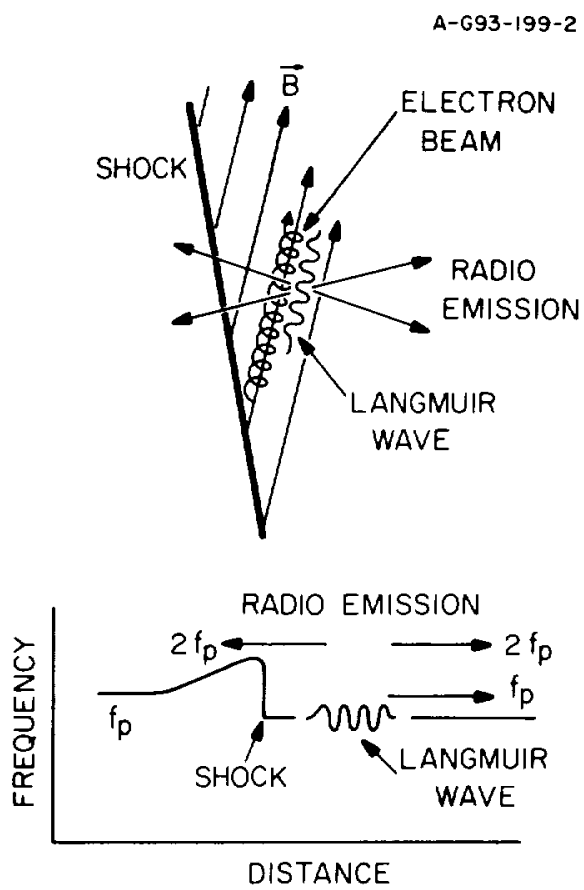

Figure 6. An illustration showing the generation of radio emission by an interplanetary shock via the Langmuir-wave mode conversion mechanism. An electron beam accelerated at the shock excites Langmuir waves, which in turn decay into electromagnetic radiation at the plasma frequency $\left(f_{p}\right)$ and at twice the plasma frequency $\left(2 f_{p}\right)$.

This location is consistent with other current estimates of the distance to the termination shock (Axford, 1996). Since the average value of $f_{p}$ at $1 \mathrm{AU}$ is about $20 \mathrm{kHz}$, the average plasma frequency at $100 \mathrm{AU}$, just ahead of the termination shock, should be about $200 \mathrm{~Hz}$. According to conventional MHD theory (Anderson, 1963), for a strong shock, which is what is expected at the termination shock, the plasma density should increase by a factor of four. Since a factor of four jump in the plasma density corresponds to a factor of two jump in the plasma frequency, the plasma frequency in the region immediately downstream of the termination shock should be about $400 \mathrm{~Hz}$. Beyond the termination shock the plasma density is expected to remain roughly constant out to the heliopause. Plasma flow simulations (Baranov and Malama, 1993; Washimi, 1993; Steinolfson et al., 1994) show that the heliopause should be located at a heliocentric distance that is about 130 to $150 \%$ of the distance to the termination shock. In Figure 7 the heliopause is therefore assumed to be at a radial distance of $140 \mathrm{AU}$. At the heliopause, a second upward jump in the plasma density is expected to occur. Since the heliopause is a contact discontinuity, the density increases by whatever factor is necessary to maintain pressure balance with the interstellar medium. Present estimates are that the electron density in the interstellar medium near the Earth is in the range from about 0.06 to $0.1 \mathrm{~cm}^{-3}$ (Lallement et al., 1993), which corresponds to a plasma frequency of about 2.2 to $2.8 \mathrm{kHz}$. Since the heliospheric bow shock is expected to be a weak shock, or may not exist at all, the plasma density immediately beyond the heliopause is expected to be very similar to the plasma density in the interstellar medium.

A number of the early papers dealing with the origin of the heliospheric $2-3 \mathrm{kHz}$ radio emission suggested that the radiation is generated upstream of the termination shock (Kurth 


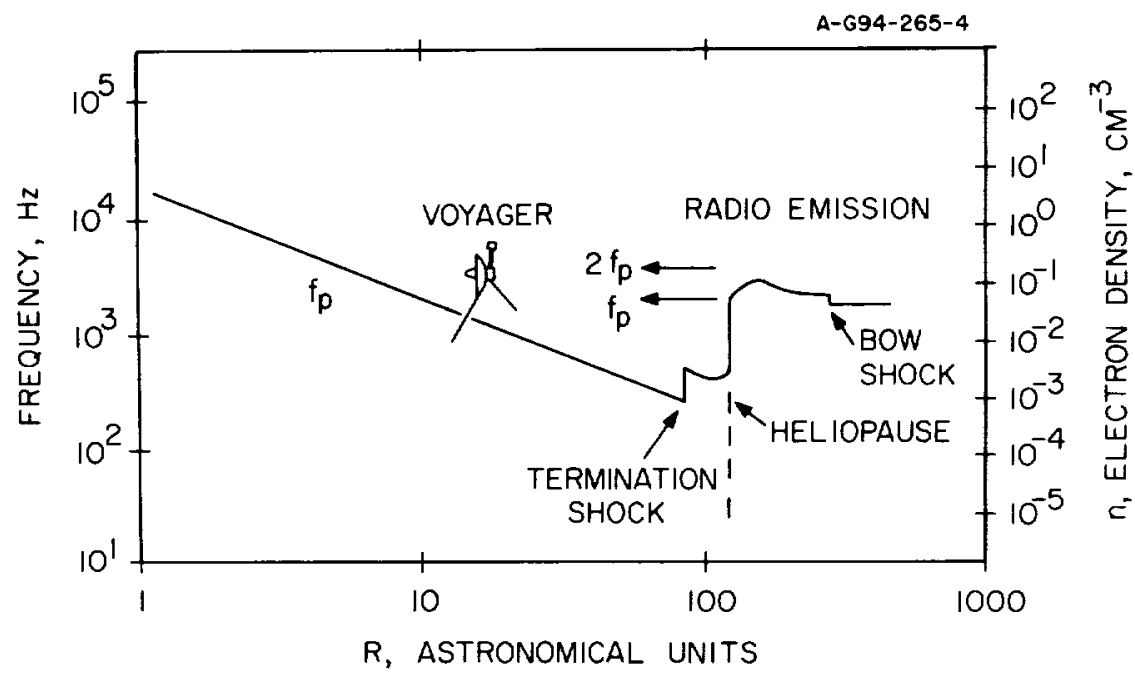

Figure 7. A model showing the plasma frequency $\left(f_{p}\right)$ as a function of the radial distance (R) from the Sun. In the supersonic solar wind the plasma frequency varies inversely with the radial distance from the Sun. At the termination shock, according to conventional MHD theory, the plasma frequency is expected to increase by a factor of two. At the heliopause, the plasma frequency increases to whatever level is necessary to maintain pressure balance with the interstellar medium.

et al., 1984; McNutt, 1988; Grzedzielski and Lazarus, 1993). From simple inspection of Figure 7, one can see that the plasma frequency upstream of the termination shock $(\sim 200$ $\mathrm{Hz}$ ) is much too low to account for the $2-3 \mathrm{kHz}$ radio emission, even if the emission is generated at $2 f_{p}$. At the heliopause, the situation is much better. Since the plasma frequency on the interstellar side of the heliopause is most likely around a few $\mathrm{kHz}$, there should be no problem generating frequencies of $2-3 \mathrm{kHz}$ in the vicinity of the heliopause. For this reason, Gurnett et al. (1993) proposed that the heliospheric $2-3 \mathrm{kHz}$ radio emission is generated in the vicinity of the heliopause.

Although the above model shows that the average plasma density in the region between the termination shock and the heliopause is too small to account for radio emission at 2-3 $\mathrm{kHz}$, a number of ideas have been suggested that could raise the emission frequency in this region. For example, Cairns et al. (1992) have pointed out that the solar wind plasma density is often strongly enhanced by transient events at the Sun. Once these density enhancements enter the region beyond the termination shock, they would not only raise the local emission frequency, but they could also potentially block the escape of radiation from regions of lower density, thereby raising the average emission frequency. In a variation of this basic idea, Zank et al. (1994) suggested that the radio emission is produced by the interaction of an interplanetary shock with density enhancements that were previously injected into the region downstream of the termination shock. Donohue and Zank (1993) have also suggested that anomalous cosmic ray pressure could alter the structure of the termination shock in such a way that the density jump exceeds the factor of four limit imposed by the usual MHD jump conditions. A larger density jump at the termination shock would raise the plasma density throughout the region downstream of the shock, thereby raising the emission frequency. Whether any of these mechanisms prove to be viable remains to be determined. 


\section{Interpretation of the Radio Emission Spectrum}

Since the emission frequency is controlled by the plasma density, the radio emission spectrum almost certainly contains valuable information on the structure of the outer heliosphere. Unfortunately, the spectrum is also quite difficult to interpret. At least three features must be explained: (1) the narrow bands that drift upward in frequency from about 2.0 to $3.6 \mathrm{kHz},(2)$ the persistent nearly constant frequency band at about $2 \mathrm{kHz}$, and (3) the sharp low-frequency cutoff at $1.8 \mathrm{kHz}$. If the radio emission is produced by an outward propagating shock, the upward drifting bands strongly suggest that the shock is propagating through a ramp of increasing plasma density. In this interpretation each upward drifting band would be caused by a different shock propagating through the same density ramp. The relatively small spread in the observed drift rates, from about 1 to $3 \mathrm{kHz}$ /year, could be easily caused by variations in the shock speeds. Using this basic idea, Gurnett et al. (1993) suggested that the upward drifting bands were produced by shocks propagating through the density pileup region that is expected to exist near the nose of the heliosphere (Steinolfson et al., 1994). In a variation of this basic mechanism, Czechowski et al. (1995) have attributed the upward drifting bands to shocks propagating through a layer of cold high-density plasma that is formed by charge exchange cooling just inside the heliopause (also see Czechowski and Grzedzielski (1995)). Zank et al. (1994) have also attributed the upward drifting bands to a shock propagating through a density ramp. However, in their model the density ramp is caused by a transient structure in the region between the termination shock and the heliopause. Suitable transient density structures could be formed either by convection of a solar wind density enhancement through the termination shock, or by another slower moving shock.

During spacecraft roll maneuvers, a well-defined intensity modulation has been observed in the upward drifting bands at $3.11 \mathrm{kHz}$ (Kurth, 1988; Gurnett et al., 1995), sometimes with intensity modulation factors as large as $60 \%$. (Due to a data system failure on Voyager 2 , roll measurements can only be made on Voyager 1). Although these one-dimensional roll modulation measurements cannot provide a unique direction to the source, the large modulation factors indicate that the angular size of the source must be relatively small. The exact size is difficult to estimate due to the unknown direction of arrival relative to the roll axis. A small source size is consistent with the relatively narrow bandwidths of the upward drifting bands, which indicate that the radio emission is generated in small isolated regions. The most likely explanation for a small isolated source is the magnetic field orientation relative to the shock front. From studies of the Earth's bow shock, it is known that the upstream electron beam is most intense for quasi-perpendicular shocks (Anderson et al., 1979). Since higher electron beam intensities most likely generate higher Langmuir wave intensities, the highest radio emission intensities are expected from regions where the magnetic field is nearly perpendicular to the shock normal. This type of magnetic control is believed to be the reason that type Il radio emissions are not generated uniformly over the shock front, but rather originate from small isolated regions (Nelson and Robinson, 1975).

The current view regarding the origin of the $2-\mathrm{kHz}$ band is that this radiation is also produced by a shock wave or a system of shock waves interacting with the outer heliosphere. To maintain the nearly constant emission frequency, the plasma frequency must be nearly constant throughout the interaction region. In contrast to the upward drifting bands, no roll modulation has ever been observed in the $2-\mathrm{kHz}$ band. The absence of roll modulation most likely means that the source subtends a very large solid angle (i.e., approaching $4 \pi$ ). These observations support the view that the radiation is generated by a quasi-spherical shock or system of shocks propagating through a region of nearly constant plasma density. Two candidate source regions are immediately evident: (1) the region between the termina- 
tion shock and the heliopause, and (2) the region beyond the heliopause. Pursuing the first possibility, Zank et al. (1994) and Whang and Burlaga (1995) both proposed that the $2-\mathrm{kHz}$ band is produced in the region between the termination shock and the heliopause. In the model of Zank et al., the shock is propagating outward from the Sun, whereas in the model of Whang and Burlaga the shock is propagating inward toward the Sun (after reflected from the heliopause). These models both suffer from the previously mentioned difficulty that the plasma frequency in the region between the termination shock and the heliopause is probably too low to account for the observed emission frequency. However, if the density jump at the termination shock exceeds the MHD limit by a substantial factor, as suggested by Donohue and Zank (1993), or if the density is enhanced by the passage of a shock, as in the model of Whang and Burlaga, then it may be possible to account for the observed emission frequency. Pursuing the second possibility, Gurnett et al. (1993) proposed that the $2-\mathrm{kHz}$ band is produced by a shock propagating through the region immediately beyond the heliopause. The plausibility of this model depends critically on the interstellar plasma density. If the plasma frequency in the interstellar medium is either about $1 \mathrm{kHz}$ (if the emission is at $2 f_{p}$ ) or about $2 \mathrm{kHz}$ (if the emission is at $f_{p}$ ), then it is a plausible model. However, if the interstellar plasma frequency is substantially above $2 \mathrm{kHz}$ (i.e., $\mathrm{n} \gtrsim 0.05$ $\mathrm{cm}^{-3}$ ), as indicated by some recent measurements, then the radiation cannot be generated in the interstellar plasma. It would then be possible for the radiation to be trapped in the low-density cavity formed by the heliosphere, as has been suggested by Czechowski and Grzedzielski (1990). Repeated reflections within the heliospheric cavity rapidly leads to a nearly isotropic intensity distribution, which would explain the absence of roll modulation. If the Q-factor of the cavity is sufficiently high (i.e., high enough to give several hundred reflections), it may also be possible to explain the long persistence time of the 2-kHz band, as well as the slow frequency drifts that are sometimes observed (Czechowski et al., 1995).

An important feature of the $2-\mathrm{kHz}$ band is the sharp, almost completely constant lowfrequency cutoff at $1.8 \mathrm{kHz}$. This cutoff could be either due to a propagation cutoff at some point between the source and the spacecraft, or to an intrinsic characteristic of the source. Because of the large density fluctuations that exist in the region between the termination shock and the heliopause, one possibility is that the density peaks act to provide an effective propagation cutoff at a frequency that is several times the average local plasma frequency (Cairns et al., 1992), thereby accounting for the cutoff at $\sim 1.8 \mathrm{kHz}$. If the propagation cutoff is controlled by the emission process, which would almost certainly be at $2 f_{p}$, then the cutoff would correspond to the minimum plasma frequency, which implies a minimum plasma frequency of $900 \mathrm{~Hz}$ (i.e., $\mathrm{n}=0.01 \mathrm{~cm}^{-3}$ ) if the source is between the termination shock and the heliopause. If the source is located beyond the heliopause, then the cutoff would most likely be caused by the propagation cutoff at the plasma frequency in the interstellar plasma. In this case the plasma density in the interstellar medium would be $0.04 \mathrm{~cm}^{-3}$, since $\mathrm{n}=0.04 \mathrm{~cm}^{-3}$ corresponds to a plasma frequency of $1.8 \mathrm{kHz}$.

\section{Conclusions}

Considerable progress has been made since the original discovery of the $2-3 \mathrm{kHz}$ radio emissions by Kurth et al. (1984). It is now known that the radio emission is caused by the interaction of an outward propagating shock, or a system of shocks, with one of the outer regions of the heliosphere, most likely in the region between the termination shock and the heliopause, or in the region near and slightly beyond the heliopause. The emissions are probably generated at the plasma frequency $\left(f_{p}\right)$ or its harmonic $\left(2 f_{p}\right)$ by the shockdriven Langmuir-wave mode conversion mechanism. A strong case can be made that the 
intense bursts observed in 1983-84 and in 1992-93 were associated with periods of intense solar activity in 1982 and 1991 . The propagation delay in both cases is about 400 days. From direct measurements of the propagation speed of the interplanetary shocks produced by these solar events, and knowledge of the time delay to the onset of the radio burst, the distance to the source can be estimated. These distances are quite large, well beyond $100 \mathrm{AU}$. Various calculations that take into account the decreasing speed of the shock with increasing distance from the Sun indicate that the radial distance to the interaction region is probably in the range from 110 to $160 \mathrm{AU}$. The radio emission spectrum is complicated and consists of three main features: (1) a series of narrow bands that drift upward in frequency from about 2.0 to $3.6 \mathrm{kHz}$ at rates ranging from about 1 to $3 \mathrm{kHz} /$ year, (2) a persistent band of emission around $2 \mathrm{kHz}$, and (3) a sharp low-frequency cutoff at $1.8 \mathrm{kHz}$. The upward drifting bands are most likely generated by a shock propagating through a ramp of rising density, either in the vicinity of the heliopause, or in association with transients density enhancements between the termination shock and the heliopause. The $2-\mathrm{kHz}$ band is probably generated by the same shock as it propagates through an extended region of nearly constant plasma frequency, either between the termination shock and the heliopause, or beyond the heliopause.

\section{Acknowledgements}

This research was supported by NASA through contract 959193 with the Jet Propulsion Laboratory.

\section{References}

Anderson, J.E.: 1963, Magnetohydrodynamic Shock Waves, MIT Press, Cambridge, 23.

Anderson, K.A., Lin, R.P., Martel, F., Lin, C.S., Park, G.K., and Reme, H.: 1979, Geophys. Res. Lett. 6, 401-404.

Axford, W.I.: 1990, in S. Grzedzielski and D.E. Page (eds.), Physics of the Outer Heliosphere, ed. by Pergamon Press, Oxford, 7-15.

Axford, W.I.: 1996, chapter in this book.

Baranov, V.B. and Malama, Yu.G.: 1993, J. Geophys. Res. 98, 15,157-15, 163.

Belcher, J.W., Lazarus, A.J., McNutt Jr., R.L. and Gordon Jr., G.S. Gordon: 1993, J. Geophys. Res. 98, 15,177-15,183.

Burlaga, L.F., McDonald, F.B. and Ness, N.F.: 1993, J. Geophys. Res. 98, 1-11.

Cairns, I.H. and Gurnett, D.A.: 1992, J. Geophys. Res. 97, 6235-6244.

Cairns, I.H., Kurth, W.S. and Gurnett, D.A.: 1992, J. Geophys. Res. 97, 6245-6259.

Cliver, E.W., Mihalov, J.D., Sheeley Jr., N.R., Howard, R.A., Koomen, M.J., and Schwenn, R.: 1987, J. Geophys. Res. 92, 8487-8501.

Czechowski, A. and Grzedzielski, S.: 1993, Nature 344, 640-641.

Czechowski, A. and Grzedzielski, S.: 1995, Adv. Space Res. 16, (9)321-(9)325.

Czechowski, A. Grzedzielski, S. and Macek, W.M.: 1995, Adv. Space Res. 16, (9)297-(9)301.

Donohue, D.J. and Zank, G.P.: 1993, J. Geophys. Res. 98, 19,005-19,025.

Dunckel, N.: 1974, Tech. Rep. 3469-2, Radioscience Laboratory, Stanford Univ., Stanford, 114-131. Gosling, J.T.: 1993, J. Geophys. Res. 98, 18,937-18,949.

Grzedzielski, S. and Lazarus, A.J.: 1993, J. Geophys. Res. 98, 5551-5558.

Gurnett, D.A. and Kurth, W.S.: 1995, Adv. Space Res. 16, (9)279-(9)290.

Gurnett, D.A., Kurth, W.S., Allendorf, S.C. and Poynter, R.L.: 1993, Science 262, 199-203.

Kurth, W.S.: 1988, in V.J. Pizzo, T.E. Holzer, and D.G. Sime (eds.), Proc. of the Sixth Internat. Solar Wind Conf., 667. 
Kurth, W.S. and Gurnett, D.A.: 1991, Geophys. Res. Lett. 18, 1801-1804.

Kurth, W.S. and Gurnett, D.A.: 1993, J. Geophys. Res. 98, 15,129-15,136.

Kurth, W.S., Gurnett, D.A., Scarf, F.L. and Poynter, R.L.: 1984, Nature 312, $27-31$.

Kurth, W.S., Gurnett, D.A., Scarf, F.L. and Poynter, R.L.: 1987, Geophys. Res. Lett. 14, 49-52.

Lallement, R., Bertaux, J.-L. and Clark, J.T.: 1993, Science 260, 1095-1098.

Liewer, P.C., Karmesin, S. Roy and Brackbill, J.U.: 1996, in D. Winterhalter, J. Gosling, S. Habbal, W. Kurth and M. Neugebauer (eds.), Proceedings of Solar Wind Eight, in press.

McDonald, F.B. and Burlaga, L.F.: 1996, in J.R. Jokipii, C.P. Sonnett and M.S. Giampapa (eds.),Cosmic Winds and the Heliosphere, Univ. of Arizona Press, in press.

McDonald, F.B., Barnes, A., Burlaga, L.F., Gazis, P., Mihalov, J. and Selesnick, R.S.: 1994, J. Geophys. Res. 99, 14,705-14,715.

McNutt, R.L., Jr.: 1988, Geophys. Res. Lett. 15, 1307-1310.

McNutt, R.L., Lazarus, A.J., Belcher, J.W., Lyon, J., Goodrich, C.C. and Kulkarni, R.: 1995, Adv. Space Res. 16, (9)303-(9)306.

Melrose, D.B.: 1985, in D.J. McLean and N.R. Labrum, Solar Radiophysics, Cambridge Univ. Press, Cambridge, 177-210.

Nelson, G.J. and Robinson, R.D.: 1975, Proc. Astron. Soc. Aust. 2, 370.

Scarf, F.L. and Gurnett, D.A.: 1977, Space Sci. Rev. 21, 289-308.

Steinolfson, R.S. and Gurnett, D.A.: 1995, Geophys. Res. Lett. 22 651-654.

Steinolfson, R.S., Pizzo, V.J. and Holzer, T.: 1994, Geophys. Res. Lett. 21, 245-248.

Van Allen, J.A.: 1993, Geophys. Res. Lett. 20, 2797-2800.

Van Allen, J.A. and Fillius, R.W.: 1992, Geophys. Res. Lett. 19, 1423-1426.

Van Allen, J.A. and Randall, B.A.: 1985, J. Geophys. Res. 90, 1399-1412.

Washimi, H.: 1993, Adv. Space Res. 6, 227-236.

Webber, W.R. and Lockwood, J.A.: 1993, J. Geophys. Res. 98, 7821-7825.

Webber, W.R., Lockwood, J.A. and Jokipii, J.R.: 1986, J. Geophys. Res. bf 91, 4103-4110.

Whang, Y.C. and Burlaga, L.F.: 1995, Adv. Space Res. (9)291-(9)295.

Winterhalter, D., Smith, E.J. and Klein, L.W.: 1992, EOS Trans. AGU 73(4), 237.

Zank, G.P., Cairns, I.H., Donohue, D.J. and Matthaeus, W.H.: 1994, J. Geophys. Res. 99, 14,72914,735 .

Address for correspondence: D. Gurnett, Dept. of Physics and Astronomy, University of Iowa, Iowa City, IA, 52242, USA 